Artículo

\title{
Presencia de enterobacterias en insumos de uso agrícola en La Comarca Lagunera
}

\author{
Claudia Zamantha Chavarría Hernández ${ }^{1}$ \\ Miguel Ángel Gallegos Robles ${ }^{2 \S}$ \\ Manuel Fortis Hernández ${ }^{3}$ \\ Uriel González Salas ${ }^{2}$ \\ María Gabriela Cervantes Vázquez ${ }^{2}$ \\ Edmundo Castellanos Pérez ${ }^{2}$
}

${ }^{1}$ Ciencias en Sustentabilidad de los Recursos Agropecuarios-UJED. Carretera Gómez Palacio-Tlahualilo km 28, Ejido Venecia, Durango. CP. 35170. Tel. 01(871) 7118918. (zamantha_chavarria@ hotmail.com). ${ }^{2}$ Facultad de Agricultura y Zootecnia- Universidad Juárez del Estado de Durango. Carretera Gómez PalacioTlahualilo km 28, Ejido Venecia, Durango. CP. 35170. Tel. 01(871) 7118918. (urgosa87@ hotmail.com; cevga@hotmail.com; ecastellmx@yahoo.com.mx). ${ }^{3}$ División de Estudios de Posgrado-Instituto Tecnológico de Torreón. Carretera Torreón-San Pedro km 7.5, Ejido Ana, Torreón, Coahuila, México. CP. 27070. Tel. (871) 7507199. (fortismanuel@ hotmail.com).

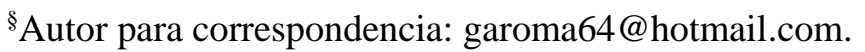

\section{Resumen}

La situación mundial del comercio de alimentos obliga a los países exportadores a reforzar sus sistemas de control y adoptar y vigilar estrategias de control de la inocuidad basadas en el riesgo de contaminación. El objetivo de esta investigación fue aislar enterobacterias en diversos insumos usados en la producción agrícola en La Comarca Lagunera y determinar el riesgo que implica su uso en las actividades agrícolas. Se analizaron microbiológicamente muestras a partir de diferentes fuentes de agua, estiércoles y suelo, usados en la producción agrícola de La Comarca Lagunera, México, durante los años 2014-2016. Se identificó a Klebsiella spp. como el principal factor de riesgo de contaminación bacteriana en el agua que se utiliza para el riego de los diferentes cultivos con una prevalencia de $46.95 \%$. En las muestras de suelo se aisló únicamente a Klebsiella spp. con una prevalencia de $60 \%$, mientras que en muestras estiércol bovino se aisló E. coli (30\%) y Klebsiella spp. (40\%), Salmonella spp. (14\%) en estiércol caprino y Salmonella spp. (100\%) en estiércol pollinaza. Lo anterior representa un factor de riesgo para la salud del consumidor final, en el caso de productores que pretendan exportar sus productos agrícolas, les restaría competitividad y les pudieran cerrar los mercados internacionales. Una investigación más profunda es necesaria a lo largo de la cadena de producción de frutas y hortalizas con el fin de reducir esta contaminación.

Palabras clave: E. coli, Klebsiella spp., Salmonella spp., inocuidad agrícola.

Recibido: abril de 2019

Aceptado: junio de 2019 


\section{Introducción}

Las frutas y vegetales proporcionan nutrientes esenciales tales como vitaminas, fibra, minerales y tienen múltiples beneficios en la salud, sin embargo, también son reconocidos como potenciales vehículos de enfermedades transmitidos por alimentos (ETA) (Mesbah et al., 2017). Actualmente se considera que para garantizar la inocuidad alimentaria de los productos agrícolas es necesario prevenir la contaminación en el campo y minimizar la contaminación cruzada durante el manejo posterior a la cosecha, sin embargo, prevenir la contaminación en campos o invernaderos es un desafío e incluso las buenas prácticas agrícolas (BPA) son insuficientes para garantizar que los patógenos humanos no se introduzcan en la cadena de productos agrícolas (Francis et al., 2012).

La producción a campo abierto de productos hortícolas los hace susceptibles a la contaminación por múltiples fuentes. Varias revisiones se han hecho sobre la fuente de contaminación con el suelo, el agua, las enmiendas biológicas y la actividad de los animales silvestres, todos considerados como rutas por las cuales se pueden introducir patógenos humanos (Warriner et al., 2009; Olaimat y Holley, 2012; Goodburn y Wallace, 2013; Nuesch-Inderbinen y Stephan, 2016).

Aún en los invernaderos, que son considerados ambientes cerrados, patógenos como Salmonella pueden llegar a establecerse (Holvoet et al., 2014). Está documentado que los patógenos entéricos pueden sobrevivir en el estiércol, el agua y el suelo durante períodos prolongados (Franz et al., 2005; Ferens y Hovde, 2011; Brandl et al., 2013) y se unen tenazmente a una variedad de semillas de plantas, raíces y hojas (Tyler y Triplett, 2008; Teplitski et al., 2011).

En México, la producción de hortalizas representa 1.6\% del valor total de la producción nacional agrícola cultivada, y en La Laguna de Coahuila y Durango equivale a 16120 millones de pesos (SIAP, 2016). En los últimos años las frutas y hortalizas frescas han llamado la atención de los investigadores no solamente por los beneficios que su consumo brinda a la salud de las personas sino también por su asociación con diversos brotes de enfermedades gastrointestinales registrados en la última década (Xuan et al., 2000). En años recientes, el origen de las enfermedades transmitidas por alimentos (ETA) ha cambiado, han surgido patógenos nuevos o cepas más agresivas y resistentes a los antibióticos (Rojas-Herrera y González-Flores, 2006).

El SENASICA promueve la implementación de sistemas de reducción de riesgos de contaminación (SRRC), con la finalidad de reducir el riesgo de contaminación en la producción de frutas y hortalizas (SAGARPA-SENASICA, 2018).

De manera que la implementación de buenas prácticas agrícolas (BPA) en las unidades de producción de las hortalizas, debería reducir los riesgos de contaminación y permitir el cumplimiento de los límites microbiológicos y de residuos de plaguicidas establecidos en la normatividad nacional e internacional. Los SRRC se han implementado en los últimos años principalmente en cultivos de exportación como chile, jitomate, cebolla y tomate (Callejas et al., 2006). Tan solo en 2016, la superficie cultivada de estas 3 hortalizas en la región lagunera fue de más de 9639.03 ha, generando un valor de producción de \$16 535 millones de pesos (SIAP, 2016). 
La Comarca lagunera es una región con gran actividad agrícola y pecuaria, y debido a la gran actividad pecuaria bovina y avícola que existe se producen anualmente 1 millón de ton de estiércol bovino y gallinaza con un gran potencial de usarse como abono orgánico en la producción agrícola (Ramírez et al., 2016). Sin embargo, estos insumos representan un alto riesgo de transmisión de agentes patógenos (Peralta-Veran et al., 2016).

De acuerdo a Sandoval y Collí (2004), otro de los factores de riesgo de contaminación microbiana a los productos hortícolas, es la práctica del riego con agua rodada procedente de afluentes naturales, de pozos contaminados y el uso de aguas residuales no tratadas. En la Región Lagunera $60 \%$ de la producción hortícola es regada con agua de noria y 40\% con agua de río (SIAP 2016). Aunque el uso de aguas residuales para riego aporta un gran número de nutrientes, algunos estudios indican que el uso de aguas no tratadas para la irrigación agrícola es la práctica que más influye en la reducción de la calidad sanitaria de frutas y hortalizas (Cazarez et al., 2004; Allende et al., 2008).

Las ETA son un problema importante a nivel mundial ya que ocasionan alta morbilidad y mortalidad, según la organización mundial de la salud, se estima que cada año se enferman en el mundo alrededor de 600 millones de personas, 1 de cada 10 habitantes por ingerir alimentos contaminados y 420000 mueren por esta misma causa (OMS, 2015). Los alimentos pueden contaminarse a lo largo de la cadena productiva y del transporte (Fatem, 2011).

Entre las bacterias causantes de ETA que son catalogadas como peligrosas se incluyen la Escherichia coli enterohemorrágica (particularmente el serovar 0157:H7), Campylobacter jejuni y Salmonella typhimurium (Blackburn y McClure, 2002). De acuerdo al CDC (2017) la bacteria Salmonella se ha encontrado en frutos de tomate, melón, calabaza y mango, mientras que E. coli O157:H7 en lechuga romana y espinacas orgánicas.

El objetivo de esta investigación fue identificar la presencia de enterobacterias en diversas fuentes de insumos usados en la producción agrícola en La Comarca Lagunera y determinar el riesgo que implica su uso en las actividades agrícolas.

\section{Materiales y métodos}

\section{Toma de muestras}

La toma de las diversas muestras se realizó en los meses de agosto a septiembre durante los años 2014-2016, en ejidos y áreas agrícolas de los municipios de San Pedro, Francisco I. Madero y Matamoros en el estado de Coahuila, y de los municipios de Lerdo y Tlahualilo en el estado de Durango (Cuadro 1).

Cuadro 1. Tipo de muestras analizadas en La Comarca Lagunera y número de muestras.

\begin{tabular}{ccc}
\hline Tipo de muestra & Localidad & $\mathrm{N}$ \\
\hline Agua de Río Nazas & Ej. León Guzmán ${ }^{*}$ & 10 \\
& Presa Francisco Zarco ${ }^{*}$ & 20 \\
Agua de pozo & Ej. Hidalgo ** & 15 \\
& Fco. I. Madero ${ }^{* *}$ & 20 \\
& Ej. Porvenir ${ }^{* *}$ & 15 \\
& Ej. Venecia & 27 \\
\hline
\end{tabular}




\begin{tabular}{|c|c|c|}
\hline Tipo de muestra & Localidad & $\mathrm{N}$ \\
\hline Agua residual & Gómez Palacio * & 20 \\
\hline \multirow[t]{16}{*}{ Agua de canal } & Ej. Hidalgo ** & 10 \\
\hline & Ej. Escuadrón ${ }^{* *}$ & 10 \\
\hline & Ej. Hormiguero ${ }^{* *}$ & 12 \\
\hline & Ej. Coyote ${ }^{* *}$ & 20 \\
\hline & Ej. 20 de noviembre ${ }^{* *}$ & 12 \\
\hline & Ej. Jaboncillo ${ }^{* *}$ & 8 \\
\hline & Ej. Santa María ${ }^{* *}$ & 7 \\
\hline & Ej. Presa de Guadalupe ${ }^{* *}$ & 8 \\
\hline & Ej. Finisterre ${ }^{* *}$ & 10 \\
\hline & Ej. Florida ${ }^{* *}$ & 10 \\
\hline & Ej. San Felipe * & 12 \\
\hline & Ej. Viñedo * & 10 \\
\hline & Ej. Venecia ${ }^{*}$ & 20 \\
\hline & Ej. Lucero * & 20 \\
\hline & Tlahualilo * & 20 \\
\hline & Matamoros ${ }^{* *}$ & 12 \\
\hline \multirow[t]{3}{*}{ Suelo (cultivo de cebolla) } & Fco. I. Madero ${ }^{* *}$ & 27 \\
\hline & Matamoros ${ }^{* *}$ & 22 \\
\hline & Ej. Hidalgo ${ }^{* *}$ & 13 \\
\hline \multirow[t]{2}{*}{ Suelo (cultivo de chile) } & Ej. Porvenir ${ }^{* *}$ & 12 \\
\hline & San Pedro de las Col. ${ }^{* *}$ & 10 \\
\hline \multirow[t]{3}{*}{ Suelo (cultivo de tomate) } & Ej. Hidalgo ${ }^{* *}$ & 20 \\
\hline & Ej. Florida ${ }^{* *}$ & 16 \\
\hline & Ej. Venecia ${ }^{*}$ & 10 \\
\hline \multirow[t]{3}{*}{ Estiércol bovino } & Fco. I. Madero ${ }^{* *}$ & 20 \\
\hline & Ej. Venecia ${ }^{*}$ & 20 \\
\hline & Ej. Lucero * & 20 \\
\hline \multirow[t]{3}{*}{ Estiércol caprino } & Ej. Florida ${ }^{* *}$ & 15 \\
\hline & Ej. Rosita ${ }^{* *}$ & 15 \\
\hline & Ej. Venecia ${ }^{*}$ & 20 \\
\hline \multirow[t]{3}{*}{ Estiércol pollinaza } & Ej. Florida ${ }^{* *}$ & 10 \\
\hline & Ej. Hidalgo ${ }^{* *}$ & 10 \\
\hline & Ej. Esfuerzo ${ }^{* *}$ & 12 \\
\hline Total & & 600 \\
\hline
\end{tabular}

${ }^{*}=$ ubicados en el estado de Durango; ${ }^{* *}=$ ubicados en el estado de Coahuila. Se recopilaron un total de 600 muestras (suelo agrícola 130, estiércol 142 y agua 328).

La metodología para la toma, manejo y transporte de muestras se basó en la NOM-230-SSA1-2002 para las muestras de agua, y en la NOM-004-SEMARNAT-2002 para las muestras de suelo y estiércol.

Las muestras para agua consistieron de $500 \mathrm{ml}$, las muestras para suelo de $500 \mathrm{~g}$ y las muestras para el estiércol de $300 \mathrm{~g}$ en fresco y $500 \mathrm{~g}$ en seco. Luego de colectadas, las muestras fueron inmediatamente almacenadas en hielo a una temperatura menor a $10{ }^{\circ} \mathrm{C}$ y transportadas al laboratorio donde se almacenaron a $4{ }^{\circ} \mathrm{C}$. Los análisis microbiológicos se realizaron dentro de las $24 \mathrm{~h}$ posteriores a la recolección de las muestras. 


\section{Análisis microbiológico}

El análisis para la presencia de coliformes fecales se llevó a cabo siguiendo la NOM-004SEMARNAT-2002. En la prueba presuntiva se pasó $1 \mathrm{ml}$ de cada una de las diluciones preparadas en tubos conteniendo caldo lactosado y se incubó por $35^{\circ} \mathrm{C} \pm 0.5{ }^{\circ} \mathrm{C}$ por $24 \pm 2 \mathrm{~h},+24 \mathrm{~h}$ en caso necesario. Los tubos positivos de la prueba presuntiva se resembraron por triple asada (esterilizada al mechero y enfriada) en tubos conteniendo caldo EC e incubados a $44.5 \pm 0.2{ }^{\circ} \mathrm{C}$ en baño de agua por $24 \pm 2 \mathrm{~h}$. El resultado fue positivo cuando hubo producción de gas a partir de la fermentación de la lactosa contenida en el medio EC.

Los tubos positivos se pasaron a agar Mac Conkey sorbitol con cefixime y telurito, se incubaron de 18 a $24 \mathrm{~h}$ a $37^{\circ} \mathrm{C}$, posteriormente se pasaron a agar TSAYE y agar EMB, y se incubaron de 24 h a $35{ }^{\circ} \mathrm{C}$. Se aislaron las colonias características de E. coli y se sometieron a las pruebas bioquímicas de indol (+), TSI (A/A + Gas), Citrato de Simmons (-), sorbitol (-), por ser sorbitol negativas. Dado que el grupo de enterobacterias incluye también a Klebsiella, ésta fue aislada en las muestras analizadas con esta metodología.

El aislamiento e identificación de Salmonella se realizó de acuerdo al protocolo descrito por Andrews et al. (2007), el cual describe brevemente, $25 \mathrm{~mL}$ ó $25 \mathrm{~g}$ de muestra se pasaron a $225 \mathrm{~mL}$ de caldo lactosado y se incubaron por $24 \mathrm{~h}$ a $35^{\circ} \mathrm{C}$. Después del periodo de incubación, las muestras se pasaron a caldo tetrationato ( $1 \mathrm{~mL}$ de muestra más $10 \mathrm{~mL}$ de caldo de tetrationato) y se dejaron incubar de 18 a $24 \mathrm{~h}$ a $42{ }^{\circ} \mathrm{C}$, después fueron sembradas en agar XLD (Becton Dickinson de México).

Estas placas se incubaron de 22 a 50 h a $35^{\circ} \mathrm{C}$. Las colonias con características de Salmonella se pasaron a pruebas bioquímicas en agar triple azúcar y hierro por sus siglas en inglés (TSI), agar hierro-lisina por sus siglas en inglés (LIA) y agar citrato de Simmons (Becton, Dickinson and Company EE. UU). La identificación del género se realizó mediante las pruebas bioquímicas de LIA, TSI y Citrato de Simmons.

Los análisis microbiológicos se realizaron en el laboratorio de microbiología de la Facultad de Agricultura y Zootecnia, ubicado en el km 28 de la carretera Gómez Palacio-Tlahualilo, Durango, en el ejido Venecia, Municipio de Gómez Palacio, Durango, México.

\section{Resultados y discusión}

\section{Análisis de muestras de agua}

El 56.09\% (184/328) de las muestras de agua (Cuadro 2) presentó contaminación con alguna enterobacteria de las más comúnmente mencionadas por la literatura (Brisse et al., 2009; PuertaGarcía y Mateos-Rodríguez, 2010). La prevalencia por microorganismo aislado fue la siguiente: Klebsiella spp. 46.95\% (154/328), Proteus spp. 8.53\% (28/328), E. coli $7.92 \%$ (26/328), Salmonella spp. 7.31\% (24/328). En este estudio se identificó a Klebsiella como el principal factor de riesgo de contaminación bacteriana en el agua que se utiliza para el riego de los diferentes cultivos en la Comarca Lagunera. Klebsiella spp. es un patógeno ubicuo y conjuntamente con $E$. coli y Enterobacter spp. son bacterias con capacidad de provocar gastroenteritis (por ingestión), infecciones respiratorias, en los oídos, la piel y otras (Rodríguez et al., 2017). 
Cuadro 2. Microorganismo aislado y prevalencia en cuatro tipos de muestras de agua usadas en el riego de cultivos en La Comarca Lagunera.

\begin{tabular}{|c|c|c|c|c|}
\hline Tipo de muestra & Localidad & $\mathrm{N}$ & Microorganismo & Prevalencia \\
\hline \multirow[t]{2}{*}{ Agua de Río Nazas } & Ej. León Guzmán * & 10 & Klebsiella & $10 / 10$ \\
\hline & Presa Francisco Zarco * & 20 & Klebsiella & $10 / 20$ \\
\hline \multirow[t]{4}{*}{ Agua de pozo } & Ej. Hidalgo ${ }^{*}$ & 15 & Proteus y & $10 / 15$ \\
\hline & Fco. I. Madero * & 20 & Klebsiella & $15 / 20$ \\
\hline & Ej. Porvenir * & 15 & Klebsiella & $15 / 15$ \\
\hline & Ej. Venecia ${ }^{*}$ & 27 & $\begin{array}{l}\text { Klebsiella } \\
\text { Salmonella }\end{array}$ & $3 / 27$ \\
\hline Agua residual & Gómez Palacio * & 20 & $\begin{array}{c}\text { E. coli y } \\
\text { Klebsiella }\end{array}$ & $20 / 20$ \\
\hline \multirow[t]{16}{*}{ Agua de canal } & Ej. Hidalgo * & 10 & Proteus y & $10 / 10$ \\
\hline & Ej. Escuadrón * & 10 & Klebsiella & $8 / 10$ \\
\hline & Ej. Hormiguero * & 12 & Proteus y & $5 / 12$ \\
\hline & Ej. Coyote ${ }^{*}$ & 20 & Klebsiella & $16 / 20$ \\
\hline & Ej. 20 de noviembre ${ }^{*}$ & 12 & Salmonella & $7 / 12$ \\
\hline & Ej. Jaboncillo ${ }^{*}$ & 8 & Klebsiella & $7 / 8$ \\
\hline & Ej. Santa María ${ }^{*}$ & 7 & Salmonella & $2 / 7$ \\
\hline & Ej. Presa de Guadalupe * & 8 & Klebsiella & $3 / 8$ \\
\hline & Ej. Finisterre ${ }^{*}$ & 10 & Klebsiella & $2 / 10$ \\
\hline & Ej. Florida ${ }^{*}$ & 10 & Klebsiella & $2 / 10$ \\
\hline & Ej. San Felipe ${ }^{* *}$ & 12 & E. coli & $3 / 12$ \\
\hline & Ej. Viñedo ${ }^{* *}$ & 10 & Salmonella & $1 / 10$ \\
\hline & Ej. Venecia ${ }^{* *}$ & 20 & Salmonella & $3 / 20$ \\
\hline & Ej. Lucero ${ }^{* *}$ & 20 & Salmonella & $15 / 20$ \\
\hline & Tlahualilo ${ }^{* *}$ & 20 & Salmonella & $13 / 20$ \\
\hline & Matamoros * & 12 & $\begin{array}{c}\text { Klebsiella } \\
\text { Klebsiella } \\
\text { E. coli }\end{array}$ & $4 / 12$ \\
\hline Total & & 328 & & $184 / 328$ \\
\hline
\end{tabular}

* = ubicados en el estado de Durango; ${ }^{* *}=$ ubicados en el estado de Coahuila.

La presencia de Klebsiella en las muestras de agua analizadas en este trabajo representa un riesgo a la salud humana y puede llegar a ser un gran problema en el control de infecciones ocasionadas por esta bacteria, dado que cepas de Klebsiella resistentes a cefalosporinas de tercera generación han sido aisladas en frutas y vegetales que se consumen en fresco (Mesbah et al., 2017) y cuya fuente de contaminación ha sido el agua de riego. Otro patógeno importante detectado en las muestras de agua fue Salmonella spp. Esta bacteria es encontrada de forma cotidiana en muestras de suelo, agua y vegetación (Puerta-García y Mateos-Rodríguez, 2010).

$\mathrm{Su}$ presencia en las muestras de agua de canales de riego representa un riesgo, dado que puede pasar a las frutas y hortalizas regadas con esta agua y de ahí al consumidor final (Goodburn y Wallace, 2013; Nuesch-Inderbinen y Stephan, 2016). Las muestras de agua que se tomaron del río Nazas presentaron una prevalencia por contaminación con enterobacterias de $66.66 \%$ (20/30), las 
muestras de agua de pozo un valor de $55.84 \%$ (43/77), las muestras de agua residual un valor del $100 \%$ (20/20) y las muestras de agua de canal un valor de 50.24\% (101/201). Estos valores son más bajos que el encontrado por Luczkiewicz et al. (2011), quienes determinaron una prevalencia de coliformes fecales de $97 \%$ en muestras de agua; sin embargo, son más altos que lo reportado por Mesbah et al. (2017) quienes encontraron una frecuencia de contaminación por enterobacterias igual a $9 \%$ en el agua de riego.

La presencia de enterobacterias en las cuatro muestras de agua analizadas en este trabajo y se usan como agua de riego en los cultivos de La Comarca Lagunera, constituye un factor de riesgo, pues existe evidencia de la contaminación de los alimentos de origen agrícola por el agua de riego y una asociación de brotes transmitidos por alimentos con vegetales, jugos y otros productos contaminados, especialmente los mínimamente procesados o de consumo en crudo (De Giglio et al., 2017).

\section{Análisis de muestras de suelo}

En los diferentes tipos de suelo (Cuadro 3) e independientemente del cultivo establecido al momento de la toma de las muestras, únicamente se aisló la bacteria Klebsiella spp. con una prevalencia de $60 \%(78 / 130)$.

Cuadro 3. Microorganismo aislado y prevalencia en tres suelos diferentes de acuerdo al cultivo establecido al momento de la toma de la muestra en La Comarca Lagunera.

\begin{tabular}{ccccc}
\hline Tipo de muestra & Localidad & N & Microorganismo & Prevalencia \\
\hline Suelo & Fco. I. Madero ${ }^{* *}$ & 27 & Klebsiella & $15 / 27$ \\
(cultivo de cebolla) & Matamoros $^{* *}$ & 22 & Klebsiella & $10 / 22$ \\
& Ej. Hidalgo $^{* *}$ & 13 & Klebsiella & $10 / 13$ \\
Suelo & Ej. Porvenir $^{* *}$ & 12 & Klebsiella & $8 / 12$ \\
(cultivo de chile) & San Pedro de las Col ${ }^{* *}$ & 10 & Klebsiella & $6 / 10$ \\
Suelo & Ej. Hidalgo $^{* *}$ & 20 & Klebsiella & $10 / 20$ \\
(cultivo de tomate) & Ej. Florida $^{* *}$ & 16 & Klebsiella & $11 / 16$ \\
& Ej. Venecia & & Klebsiella & $8 / 10$ \\
Total & 10 & & $78 / 130$ \\
\hline
\end{tabular}

$*$ ubicados en el estado de Durango. ${ }^{* *}=$ ubicados en el estado de Coahuila.

En el suelo donde estaba establecido la cebolla se observó una prevalencia de $56.45 \%$ (35/62), para el suelo donde estaba establecido cultivo de chile una prevalencia de $63.63 \%$ (14/22) y para el suelo donde había cultivo de tomate se observó una prevalencia de 63\% (29/46). Klebsiella spp. es encontrada en el ambiente de forma cotidiana, y el suelo no es la excepción. Rossmann et al. (2012) describieron la incidencia de Klebsiella spp. en $11.4 \%$ en la rizósfera del suelo y también reportaron la presencia de enterobacterias con potencial patógeno humano con menos prevalencia tales como Salmonella y E. coli. En este estudio no se detectó Salmonella ni E. coli en las muestras de suelo.

\section{Análisis de muestras de estiércol}

Respecto a las enterobacterias aisladas en los tres tipos de estiércol (Cuadro 4), la prevalencia fue de $41.54 \%$ (59/142). La prevalencia por tipo de estiércol fue la siguiente: bovino 33.3\% (20/60), caprino $14.0 \%(7 / 50)$ y pollinaza $100 \%(32 / 32)$. 
Cuadro 4. Microorganismo aislado y prevalencia en tres tipos diferentes de estiércol en La Comarca Lagunera.

\begin{tabular}{ccccc}
\hline Tipo de muestra & Localidad & N & Microorganismo & Prevalencia \\
\hline \multirow{3}{*}{ Estiércol bovino } & Fco. I. Madero ${ }^{* *}$ & 20 & E. coli & $6 / 20$ \\
& Ej. Venecia $^{*}$ & 20 & E. coli & $6 / 20$ \\
& Ej. Lucero $^{*}$ & 20 & Klebsiella & $8 / 20$ \\
Estiércol caprino & Ej. Florida $^{* *}$ & 15 & Salmonella spp. & $3 / 15$ \\
& Ej. Rosita $^{* *}$ & 15 & Salmonella spp. & $4 / 15$ \\
& Ej. Venecia $^{*}$ & 20 & Salmonella spp. & $0 / 20$ \\
Estiércol pollinaza & Ej. Florida $^{* *}$ & 10 & Salmonella spp. & $10 / 10$ \\
& Ej. Hidalgo $^{* *}$ & 10 & Salmonella spp. & $10 / 10$ \\
Total & Ej. Esfuerzo & & & \\
& & 12 & Salmonella spp. & $12 / 12$ \\
& & 142 & & $59 / 142$ \\
\hline
\end{tabular}

* = ubicados en el estado de Durango; ${ }^{* *}=$ ubicados en el estado de Coahuila.

En el estiércol bovino se aisló E. coli y Klebsiella spp., mientras que en el estiércol caprino y pollinaza únicamente se aisló Salmonella spp. Lo anterior, constituye un fuerte factor de riesgo para la salud humana, debido a que el uso de antibióticos para tratar humanos y animales en la agricultura puede llevar a la selección de bacterias resistentes a los antibióticos que se escapan al medio ambiente en los estiércoles (Durso y Cook, 2014).

Los aislamientos ambientales de enterobacterias que han adquirido resistencia a las cefalosporinas de tercera generación (3GC) constituyen una amenaza crucial para la salud pública como fuentes de resistencia para cepas bacterianas patógenas que podrían conducir a un fracaso en la antibioterapia (Blaak et al., 2014).

La presencia de residuos en los alojamientos de los cerdos, vacas, pollos, cabras ha sido identificada como un importante factor de riesgo para la infección por Salmonella (Beloeil et al., 2004). Salmonella puede sobrevivir durante meses en restos de polvo y materia orgánica presentes en los corrales, equipamientos y sistemas de ventilación (Rajic et al., 2005), estando esta supervivencia marcada por algunos factores como el serotipo y las condiciones climáticas. Por ello, es de suma importancia emplear protocolos de limpieza y desinfección eficaces en los establos.

Diversos estudios han probado que la aplicación de un protocolo adecuado de limpieza y desinfección es una medida importante para prevenir la infección por Salmonella dentro de los establos (Schmidt et al., 2004; Hautekiet et al., 2008).

En el caso de los tres tipos de estiércol analizados (Cuadro 3), se observa que la contaminación por Salmonella se encuentra en la pollinaza y no el estiércol de vaca, posiblemente debido a que la región es una cuenca lechera importante, lo que puede indicar que en los establos bovinos hay buenas prácticas de manejo de excretas. El desarrollo de diagnóstico para bacterias enteropatógenas, asociadas con productos hortícolas permitirá establecer medidas más adecuadas de control en la cadena de producción al identificar las fuentes de contaminación como el agua de riego, estiércol, suelo con tendencia al cultivo hortícola, hacia las cuales se ha dirigido la investigación. 
Los resultados encontrados en esta investigación resaltan la importancia de darle al estiércol un tratamiento tendiente a minimizar la carga de microrganismos patógenos antes de usarse como abono en la producción agrícola, ya que está demostrado que además de mejorar la fertilidad del suelo, también son portadores de patógenos zoonoticos que tienen el potencial de causar enfermedades en el humano (Hutchison et al., 2005).

\section{Conclusiones}

Los insumos evaluados en este trabajo y que son aplicados en la producción agrícola en La Comarca Lagunera representan un factor de riesgo para el consumidor por la presencia de enterobacterias. Lo anterior, además de ser una fuente de enfermedades trasmitidas por alimentos, resta competitividad a los productores para vender sus productos agrícolas. Adicionalmente se debería informar al consumidor final acerca de estos peligros microbiológicos para la salud y fomentar las buenas prácticas de higiene doméstica. Se deduce también que más investigación es necesaria a lo largo de la cadena de producción de frutas y hortalizas con el fin de involucrar más profesionales en inocuidad agrícola en acciones para reducir esta contaminación.

\section{Agradecimientos}

A la Universidad Juárez del Estado de Durango por el apoyo económico para realizar la investigación y al CONACYT por la beca otorgada para concluir mis estudios de doctorado.

\section{Literatura citada}

Allende, A.; Selma, M. V.; López-Golvez, F.; Villaescusa, R. and Gil, M. I. 2008. Role of commercial sanitizers and washing systems on epiphytic microorganisms and sensory quality of fresh-cut escarole and lettuce. Postharvest Biol. Technol. 49(1):155-163.

Andrews, W.; Wang, H.; Jacobson, A. and Hammack, T. 2007. Bacteriological analytical manual. Chapter 5. Salmonella. https://www.fda.gov/food/foodscienceresearch/laboratorymethods/ ucm070149.htm.

Beloeil, P. A.; Fravalo, P.; Fablet C.; Jolly, J. P.; Eveno, E.; Hascoet, Y.; Chauvin, C.; Salvat, G. and Madec, F. 2004. Risk factors for Salmonella enterica subsp. enterica shedding by market-age pigs in French farrow-to-finish herds. Prev. Vet. Med. 63(1-2):103-120.

Blaak, H.; van Hoek, A. H.; Veenman, C.; van Leeuwen, A. E.; Lynch, G.; van Overbeek, W. M.; de Roda Husman, A. M. 2014. Extended spectrum B-lactamase- and constitutively AmpCproducing Enterobacteriaceae on fresh produce and in the agricultural environment. Int. J. Food Microbiol. (168-169):8-16. doi: 10.1016/j.ijfoodmicro.2013.10.006.

Blackburn, C. and McClure, P. J. 2002. Foodborne pathogens: hazards, risk analysis and control. CRC Press. Boca Raton, FL. USA. 521 p.

Brandl, M. T.; Cox, C. E.; Teplitski, M. 2013. Salmonella interactions with plants and their associated microbiota. Phytopathology. 103(4):316-325.

Brisse, S.; Fevre, C.; Passet, V.; Issenhuth-Jeanjean, S.; Tournebize, R.; Diancourt, L. and Grimont, P. 2009. Virulent clones of Klebsiella pneumoniae: identification and evolutionary scenario based on genomic and phenotypic characterization. PLoS ONE. 4(3):1-13.

Callejas, J. N.; Matus-Gardea, J. A.; García-Salazar, J. A.; Martínez-Damián, M. Á. y SalasGonzález, J. Ma. 2006. Situación actual y perspectivas de mercado para la tuna, el nopalito y derivados en el Estado de México. Agrociencia. 43(1):73-82. 
Cazárez, G.; Gortares, P.; Rubio, W.; Martínez, C.; Meza, P. y Chaidez, C. 2004. Presencia y supervivencia de coliformes fecales, Salmonella spp. y Listeria spp. en agua de uso agrícola en el valle de Culiacán. In: XIV Congreso Nacional. Federación Mexicana de Ingeniería Sanitaria y Ciencias Ambientales. Mazatlán, Sinaloa. 12 al 14 de mayo.

CDC. Center of Disease Control. 2017. Foodborne Illness and Outbreaks. https://www.cdc.gov/ foodsafety/outbreaks/multistate-outbreaks/outbreaks-list.html.

De Giglio, O.; Caggiano, G.; Bagordo, F.; Barbuti, G.; Brigida, S.; Lugoli, F.; Grassi, T.; La Rosa, G.; Lucentini, L.; Uricchio, V. F.; De Donno, A. and Montagna, M. T. 2017. Enteric viruses and fecal bacteria indicators to assess groundwater quality and suitability for irrigation. Int. J. Environ. Res. Public Health. 14(6):2-13.

Durso, L. M. and Cook, K. L. 2014. Impacts of antibiotic use in agriculture: what are the benefits and risks? Curr. Opin. Microbiol. 19(1):37-44.

Fatem, M. 2011. The incidence of Enterobacteriaceae causing food poisoning in some meat products. Adv. J. Food Sci. Technol. 3(2):116-121.

Ferens, W. A. and Hovde, C. J. 2011. Escherichia coli O157:H7: animal reservoir and sources of human infection. Foodborne Pathog. Dis. 8(4):465-87.

Francis, G. A.; Gallone, A.; Nychas, G. J.; Sofos, J. N.; Colelli, G.; Amodio, M. L. and Spano, G. 2012. Factors affecting quality and safety of fresh-cut produce. Crit. Rev. Food Sci. Nutr. 52(7):595-610.

Franz, E.; van Diepeningen, A. D.; De Vos, O. J. and van Bruggen, A. H. C. 2005. Effects of cattle feeding regimen and soil management type on the fate of Escherichia coli O157:H7 and Salmonella enterica serovar typhimurium in manure, manure-amended soil, and lettuce. Appl. Environ. Microbiol. 71(10):6165-6174.

Goodburn, C. and Wallace, C. A. 2013. The microbiological efficacy of decontamination methodologies for fresh produce: A review. Food Control. 32(2):418-427.

Hautekiet, V.; Geert, V.; Marc, V. and Rony, G. 2008. Development of a sanitary risk index for Salmonella seroprevalence in Belgian pig farms. Prev. Vet. Med. 86(1-2):75-92.

Holvoet, K.; Sampers, I.; Seynnaeve, M.; Jacxsens, L. and Uyttendaele, M. 2014. Agricultural and management practices and bacterial contamination in greenhouse versus open field lettuce production. Int. J. Environ. Res. Public. Health. 12(1):32-63.

Hutchison, M.; Walters, L.; Moore, T.; Thomas, D.; and Avery, S. 2005. Fate of pathogens present in livestock wastes spread onto fescue plots. Appl. Environ. Microbiol. 71(2):691-696.

Luczkiewicz, A.; Jankowska, K.; Kurlenda, J.; Olanczuk-Neyman, K. 2011. Identification and antimicrobial susceptibility of fecal coliforms isolated from surface water. Polish J. Environ. Stud. 20(4):941-950.

Mesbah, Z. F.; Granier, S. A.; Marault, M.; Yaici, L.; Gassilloud, B.; Manceau, C.; Touati, A. and Millemann, Y. 2017. From farms to markets: gram-negative bacteria resistant to third generation Cephalosporins in fruits and vegetables in a region of North Africa. Front. Microbiol. 8:1569. doi:10.3389/fmicb.2017.01569.

NOM-004-SEMARNAT-2002. Protección ambiental. -lodos y biosólidos. -especificaciones y límites máximos permisibles de contaminantes para su aprovechamiento y disposición final. http://dof.gob.mx/nota-detalle.php?codigo=691939\&fecha=15/08/2003.

NOM-230-SSA1-2002. Salud ambiental. Agua para uso y consumo humano, requisitos sanitarios que se deben cumplir en los sistemas de abastecimiento públicos y privados durante el manejo del agua. Procedimientos sanitarios para el muestreo. http://www.salud.gob.mx/ unidades/cdi/nom/230ssa102.html. 
Nuesch-Inderbinen, M. and Stephan, R. 2016. Fresh fruit and vegetables as vehicles of bacterial foodborne disease: a review and analysis of outbreaks registered by proMED-mail associated with fresh produce. J. Food Saf. Food Qual. 67(2):32-39.

Olaimat, A. N. and Holley, R. A. 2012. Factors influencing the microbial safety of fresh produce: a review. Food Microbiol. 32(1):1-19.

Peralta-Veran, L.; Juscamaita-Morales, J. y Meza-Contreras, V. 2016. Obtención y caracterización de abono orgánico líquido a través del tratamiento de excretas del ganado vacuno de un establo lechero usando un consorcio microbiano ácido láctico. Ecol. Apl. 15(1):1-10.

Puerta-García, A. y Mateos-Rodríguez, F. 2010. Enterobacterias. Medicine. 10(51):3426-3431.

Rajic, A.; Keenliside, J.; McFall, M.; Deckert, A.; Muckle, A.; O'Connor, B.P.; Manninen, K.; Dewey, C. and McEwen, S. 2005. Longitudinal study of Salmonella species in 90 Alberta swine finishing Farms. Vet. Microbiol. 105(1):47-56.

Ramírez, J. A.; Figueroa, U.; Núñez, G.; Reta, D. G. y García, J. L. 2016. Evaluation of tillage methods and manure incorporation into corn silage production. RChSZA. 15(2):67-76.

Rodríguez, R.; Retamozo-Chavez, R.; Aponte, H. y Valdivia, E. 2017. Evaluación microbiológica de un cuerpo de agua del ACR humedales de ventanilla (Callao, Perú) y su importancia para la salud pública local. Ecología Aplicada. 16(1):15-21.

Rojas-Herrera, R. A. y González-Flores, T. 2006. Detección e identificación de bacterias causantes de enfermedades transmitidas por alimentos mediante la reacción en cadena de la polimerasa. Bioquimia. 31(2):69-76.

Rossmann, B.; Müller, H.; Smalla, K.; Mpiira, S.; Baptist, J.; Staver, Ch. and Berg, G. 2012. Banana-associated microbial communities in uganda are highly diverse but dominated by Enterobacteriaceae. Appl. Environ. Microbiol. 78(14):4933-4941.

SAGARPA-SENASICA. 2018. Secretaría de Agricultura, Ganadería, Desarrollo Rural, Pesca y Alimentación-Servicio Nacional de Sanidad-Inocuidad y Calidad Agroalimentaria. Guía de Evaluación de los SRRC. https://www.gob.mx/senasica/documentos/guias-para-laevaluacion-de-los-srrc.

Sandoval, L. y Collí, J. 2004. Tratamiento integral de agua residual municipal, su desinfección y reuso en la agricultura. In: XXIX Congreso Interamericano de Ingeniería Sanitaria y Ambiental. San Juan, Puerto Rico.

Schmidt, P. L.; O'Connor, A. M.; McKean, J. D. and Hurd, H. S. 2004. The association between cleaning and desinfection of lairage pens and the prevalence of Salmonella enterica in swine harvest. J. Food Prot. 67(7):1384-1388.

SIAP. 2016. Servicio de Información Agroalimentaria y Pesquera. Cierre de la producción agrícola. Nopalitos. http://www.siap.gob.mx.

Teplitski, M.; Warriner, K.; Bartz, J. and Schneider, K. R. 2011. Untangling metabolic and communication networks: interactions of enterics with phytobacteria and their implications in produce safety. Trends. Microbiol. 19(3):121-127.

Tyler, H. L. and Triplett, E. 2008. Plants as a habitat for beneficial and human pathogenic bacteria. Annu. Rev. Phytopathol. 46:53-73.

Warriner, K.; Huber, A.; Namvar, A.; Fan, W. and Dunfield, K. 2009. Recent advances in the microbial safety of fresh fruits and vegetables. Adv. Food Nutr. Res. 57:155-208.

Xuan, G.; Chen, J.; Beuchat, L. R. and Brackett, R. E. 2000. PCR detection of Salmonella enterica serotype Montevideo in and on raw tomatoes using primers derived from hilA. Appl. Environ. Microbiol. 66(12):5248-5252. 